\title{
The effect of posterior polyester tethers on the biomechanics of proximal junctional kyphosis: a finite element analysis
}

\author{
Shay Bess, MD, ${ }^{1}$ Jeffrey E. Harris, MS, ${ }^{2}$ Alexander W. L. Turner, $\mathrm{PhD},{ }^{2}$ Virginie LaFage, $\mathrm{PhD},{ }^{3}$ \\ Justin S. Smith, MD, PhD, ${ }^{4}$ Christopher I. Shaffrey, MD, ${ }^{4}$ Frank J. Schwab, MD, ${ }^{3}$ and \\ Regis W. Haid Jr., MD ${ }^{5}$
}

'Department of Orthopaedic Surgery, New York University/Hospital for Joint Diseases; ${ }^{3}$ Department of Orthopaedic Surgery, Hospital for Special Surgery, New York, New York; ${ }^{2}$ NuVasive, Inc., San Diego, California; ${ }^{\circledR}$ Department of Neurosurgery, University of Virginia Medical Center, Charlottesville, Virginia; and ${ }^{5}$ Atlanta Brain and Spine Care, Atlanta, Georgia

OBJECTIVE Proximal junctional kyphosis (PJK) remains problematic following multilevel instrumented spine surgery. Previous biomechanical studies indicate that providing less rigid fixation at the cranial aspect of a long posterior instrumented construct, via transition rods or hooks at the upper instrumented vertebra (UIV), may provide a gradual transition to normal motion and prevent PJK. The purpose of this study was to evaluate the ability of posterior anchored polyethylene tethers to distribute proximal motion segment stiffness in long instrumented spine constructs.

METHODS A finite element model of a T7-L5 spine segment was created to evaluate range of motion (ROM), intradiscal pressure, pedicle screw loads, and forces in the posterior ligament complex within and adjacent to the proximal terminus of an instrumented spine construct. Six models were tested: 1) intact spine; 2) bilateral, segmental pedicle screws (PS) at all levels from T-11 through L-5; 3) bilateral pedicle screws from T-12 to L-5 and transverse process hooks (TPH) at T-11 (the UIV); 4) pedicle screws from T-11 to L5 and 1-level tethers from T-10 to T-11 (TE-UIV+1); 5) pedicle screws from T-11 to L-5 and 2-level tethers from T-9 to T-11 (TE-UIV+2); and 6) pedicle screws and 3-level tethers from T-8 to T-11 (TE-UIV+3).

RESULTS Proximal-segment range of motion (ROM) for the PS construct increased from $16 \%$ at UIV-1 to $91 \%$ at UIV. Proximal-segment ROM for the TPH construct increased from $27 \%$ at UIV-1 to $92 \%$ at UIV. Posterior tether constructs distributed ROM at the UIV and cranial adjacent segments most effectively; ROM for TE-UIV+1 was $14 \%$ of the intact model at UIV-1, 76\% at UIV, and $98 \%$ at UIV+1. ROM for TE-UIV+2 was $10 \%$ at UIV-1, $51 \%$ at UIV, $69 \%$ at UIV+1, and $97 \%$ at UIV+2. ROM for TE-UIV+3 was $7 \%$ at UIV-1, $33 \%$ at UIV, $45 \%$ at UIV+1, and $64 \%$ at UIV+2. Proximal segment intradiscal pressures, pedicle screw loads, and ligament forces in the posterior ligament complex were progressively reduced with increasing number of posterior tethers used.

CONCLUSIONS Finite element analysis of long instrumented spine constructs demonstrated that posterior tethers created a more gradual transition in ROM and adjacent-segment stress from the instrumented to the noninstrumented spine compared with all PS and TPH constructs. Posterior tethers may limit the biomechanical risk factor for PJK; however, further clinical research is needed to evaluate clinical efficacy.

http://thejns.org/doi/abs/10.3171/2016.6.SPINE151477

KEY WORDS biomechanics; proximal junctional kyphosis; spine deformity

ABBREVIATIONS ISL = interspinous ligament; PJF = proximal junctional failure; $\mathrm{PJK}=$ proximal junctional kyphosis; $\mathrm{ROM}=$ range of motion; $\mathrm{SSL}=$ supraspinous ligament; TPH = transverse process hooks; UIV = upper instrumented vertebra.

SUBMITTED December 21, 2015. ACCEPTED June 21, 2016.

INCLUDE WHEN CITING Published online September 9, 2016; DOI: 10.3171/2016.6.SPINE151477. 
$\mathrm{P}$ ROXIMAL junctional kyphosis (PJK) is a common postoperative complication following multilevel instrumented surgery for spinal deformity. ${ }^{9,14,15,28}$ PJK is defined as focal kyphosis involving the vertebral segments proximal to the upper instrumented vertebra (UIV) in which: 1) the segmental kyphosis between the UIV and the vertebra that is 2 levels cranial to the UIV $(\mathrm{UIV}+2)$ is $10^{\circ}$ or greater and 2) the postoperative segmental kyphosis between UIV and UIV+2 (UIV/UIV+2 segmental kyphosis) is at least $10^{\circ}$ greater than the preoperative value. ${ }^{9}$ Despite the relatively frequent occurrence of PJK, the clinical significance of PJK remains debatable. Some reports indicate that PJK is only a radiographic phenomenon with little or no clinical impact, whereas others indicate that PJK can be associated with pain, neurological deficit, and the need for revision spine surgery. ${ }^{2,12,13,29}$ However, a recent retrospective review of the causes for hospital readmission following adult spinal deformity surgery reported that PJK was the most common postoperative complication that required surgical treatment in a cohort of 836 adult spinal deformity patients. ${ }^{21}$

Risk factors for developing PJK have been identified, including patient age, poor bone quality, posterior spinal ligament disruption, spinal instrumentation construct rigidity, fusion to the sacrum, and postoperative spinal alignment. ${ }^{5,6,14,15}$ While the causes of PJK are likely multifactorial, one theory is that PJK is in part due to a biomechanical phenomenon secondary to an abrupt change in rigidity between the nonmobile instrumented vertebral segments and the adjacent relatively hypermobile noninstrumented segments. Consequently, there have been efforts to mitigate the biomechanical risk factor for PJK by reducing the rigidity of the instrumentation at the UIV via the use of transverse process hooks or tapered transition rods..$^{4,5,7,25}$ Consistent with this work, it is possible that augmentation of the posterior spinal ligament complex using elastic tethers anchored at the UIV and above will provide another method to allow for a more gradual transition from the rigid instrumented construct to the uninstrumented mobile segments.

The purpose of this study was to evaluate the effect posterior column polyester tethers have on spinal range of motion, intradiscal pressures, and pedicle screw loads using finite element models of multilevel instrumented spinal constructs, thereby evaluating whether posterior tethers provide a viable gradual transition to normal motion over multiple spinal segments above the UIV. Finite element models were used rather than cadaveric testing due to limitations of accurately measuring and comparing construct loads and motions in a cadaver model.

\section{Methods}

\section{Finite Element Model}

A 3-dimensional finite element model of a T7-L5 spine segment was developed using geometry from a morphologically correct spine model that included the vertebrae and intervertebral discs (Zygote Media Group, Inc.). The rib cage was excluded from the analysis because of the difficulty and complexity of accurately representing the stiffness and external muscle forces due to the ribs. The geometrical surfaces of the spine model were imported into Hypermesh (Altair) and meshed in a combination of tetrahedral elements for the vertebrae and hexahedral elements for the intervertebral discs. The vertebrae were modeled with a cortical shell and cancellous core. The intervertebral discs, comprising the annulus fibrosus and nucleus pulposus, were represented using a hyperelastic Mooney-Rivlin formulation, which approximates the incompressible nature of the intervertebral disc. ${ }^{23}$ The major spinal ligaments represented in the model were the anterior longitudinal ligament, posterior longitudinal ligament, ligamentum flavum, interspinous ligament (ISL), supraspinous ligament (SSL), and capsular ligaments. Ligaments were modeled using published experimental values and approximated as nonlinear, tension-only springs (ANSYS 16.0) with insertion points approximated to typical anatomy. ${ }^{23}$ The material properties of each anatomical structure in the model were taken from published literature and are shown in Table 1.,10,17,23 The material properties of the elastic tether were obtained experimentally on a bench-top test and the nonlinear force-displacement relationships measured in the test were represented in the model as tensiononly, nonlinear springs. The elastic properties of the tethers were validated by comparing the force-displacement curves in the model to those found experimentally. The connection of the tethers to pedicle screws was approximated as a remote attachment between the tether and head of the pedicle screw shank.

\section{Test Conditions}

Because PJK is considered a forward decompensation of the spine and tethers primarily restrict flexion moments, only flexion tests were performed. An unconstrained pure flexion moment of $5.0 \mathrm{~N} \times \mathrm{m}$ was applied to the superior endplate of T-7. The distal vertebra was restricted from all motion by rigidly anchoring the inferior endplate of L-5, effectively acting as a fusion to the pelvis. A pure moment load was used because of 2 primary advantages: a pure moment load is independent of spinal geometry, as the applied moment on the proximal vertebrae is applied equally to all segments in the spine, and the pure moment remains unchanged as the spine deforms during testing. ${ }^{19,20}$ Six spine models were evaluated, an intact spine model and 5 instrumented spine models (Fig. 1):

1) Intact model-intact spine without instrumentation, to be used as a baseline comparator.

2) PS model-a standard construct with bilateral, segmental pedicle screw instrumentation at all levels and 5.5mm-diameter cobalt-chromium rods from T-11 to L-5.

3) TPH model-transverse process hooks at the UIV, with bilateral pedicle screws from T-12 to L-5 and hooks at T-11.

4) TE-UIV+1-one-level posterior tethers anchored to the $\mathrm{UIV}+1$ (tether extending from T-11 to T-10).

5) TE-UIV+2 - two-level tethers with separate tethers anchored onto UIV+1 and UIV+2 (tethers extending from T-11 to T-10 and T-9).

6) TE-UIV+3 - three-level tethers anchored onto UIV+1, $\mathrm{UIV}+2$ and $\mathrm{UIV}+3$ (tethers extending from $\mathrm{T}-11$ to T-10, T-9, and T-8). 
TABLE 1. Summary of material properties for anatomical structures in finite element model

\begin{tabular}{|c|c|c|c|}
\hline Property & Modulus (MPa) & $v$ & References \\
\hline Cortical bone & 12,000 & 0.2 & Goel et al., 1995 \\
\hline $\begin{array}{l}\text { Cancellous } \\
\text { bone }\end{array}$ & 300 & 0.2 & $\begin{array}{l}\text { Morgan et al., 2003; } \\
\text { Bowden et al., } 2008\end{array}$ \\
\hline $\begin{array}{l}\text { Annulus } \\
\text { fibrosus }\end{array}$ & $\begin{array}{l}\text { Mooney-Rivlin } \\
c_{1}=0.18, c_{2}=0.045\end{array}$ & NA & Schmidt et al., 2007 \\
\hline $\begin{array}{l}\text { Nucleus pul- } \\
\text { posus }\end{array}$ & $\begin{array}{l}\text { Mooney-Rivlin } \\
c_{1}=0.12, c_{2}=0.03\end{array}$ & NA & Schmidt et al., 2007 \\
\hline Ligaments & Hyperelastic & NA & Schmidt et al., 2007 \\
\hline
\end{tabular}

$\mathrm{NA}=$ not applicable

\section{Model Validation}

The stiffness of each spine segment in the major motion directions (flexion-extension, lateral bending, and axial rotation) is primarily controlled by the material properties of the spinal ligaments and intervertebral disc.,11 To validate the intact/uninstrumented spine model, segmental stiffness was compared with experimental in vitro results from the literature..$^{8,18,22-24}$ Because the boundary conditions varied between studies, rotational segment stiffness was used to enable equivalent comparisons. The results from the finite element model were within ranges reported in the literature.

\section{Data Analysis}

Intervertebral range of motion (ROM) for the segments between T-7 and T-12, intradiscal pressure, pedicle screw forces, and ligament forces in the ISL/SSL ligament complex were measured. Segmental ROM was determined by measuring the change in angle between the superior and inferior endplate of each spinal motion segment. Intradiscal pressures were determined by averaging the pressure of the elements in the center of the nucleus pulposus. Pedicle screw forces were determined by measuring the contact reaction forces at the bone-screw interface. Forces in the ISL/SSL complex were determined from the forcedisplacement relationship defined in the ligament material properties. Segmental ROM, intradiscal pressure, and ISL/SSL ligament complex forces were normalized to values for the intact spine.

\section{Results}

\section{Segmental Range of Motion}

The ROM at the UIV/UIV+1 segment for the pedicle screw model abruptly increased from $16 \%$ of intact ROM at T11-12 to $91 \%$ of intact ROM at T10-11. Transitional $\mathrm{ROM}$ at the UIV/UIV+1 segment for the TPH model demonstrated a more gradual transition, as T11-12 ROM was $27 \%$ of intact, with ROM increasing to $92 \%$ of intact at T10-11. Modeling of the posterior tether constructs demonstrated further dampening, as transitional ROM for the TE-UIV+1 model was $14 \%$ at $\mathrm{T} 11-12,76 \%$ at $\mathrm{T} 10-11$, and 98\% at T9-10. Transitional ROM for TE-UIV+2 model was $10 \%$ at $\mathrm{T} 11-12,51 \%$ at $\mathrm{T} 10-11,69 \%$ at $\mathrm{T} 9-10$, and 97\% at T8-9. Transitional ROM for TE-UIV+3 was 7\% at $\mathrm{T} 11-12,33 \%$ at $\mathrm{T} 10-11,45 \%$ at $\mathrm{T} 9-10,64 \%$ at $\mathrm{T} 8-9$, and $97 \%$ at T7-8 (Fig. 2).

\section{Intradiscal Pressure}

Evaluation of intradiscal pressure for the PS model demonstrated that the change in intradiscal pressure abruptly increased from $34 \%$ of intact at T11-12 to $100 \%$

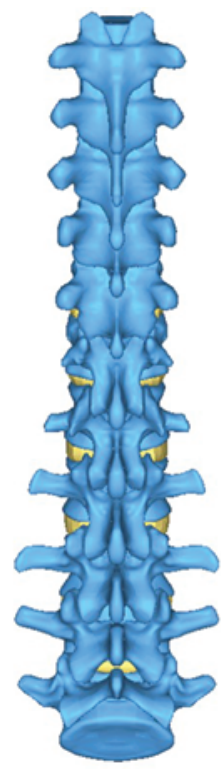

Intact

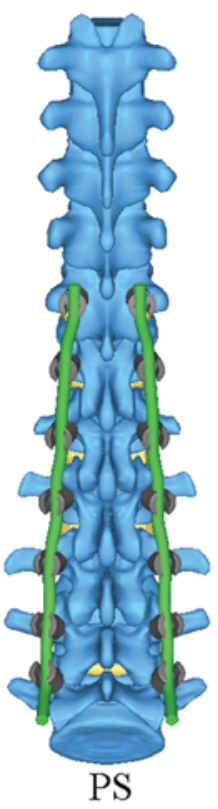

PS

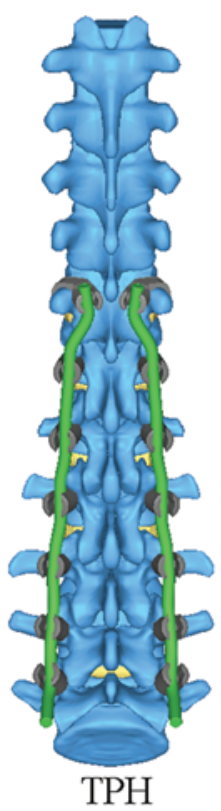

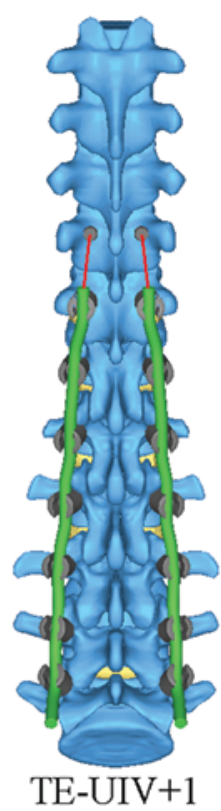

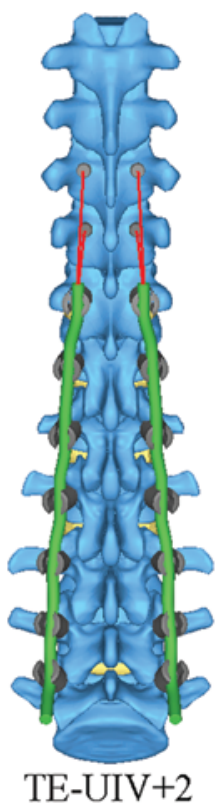

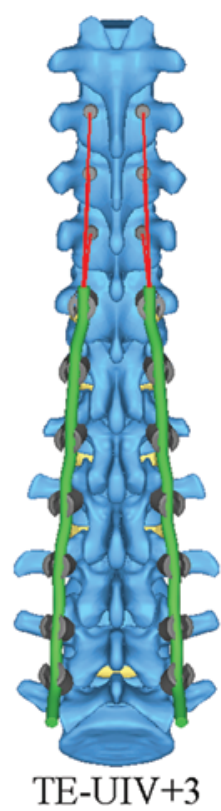

FIG. 1. Posterior view of spine models tested: intact; with bilateral segmental pedicle screws and rods from T-11 to L-5 (PS); transverse process hooks at T-11 with segmental, bilateral pedicle screws from T-12 to L-5 (TPH); 1-level tether anchored from the UIV to UIV+1 (TE-UIV+1); 3-level tether anchored from the UIV to UIV+1 and UIV+2 (TE-UIV+2); 3-level tethers anchored from the UIV to $U I V+1, U I V+2$, and $U I V+3(T E-U I V+3)$. Figure is available in color online only. 


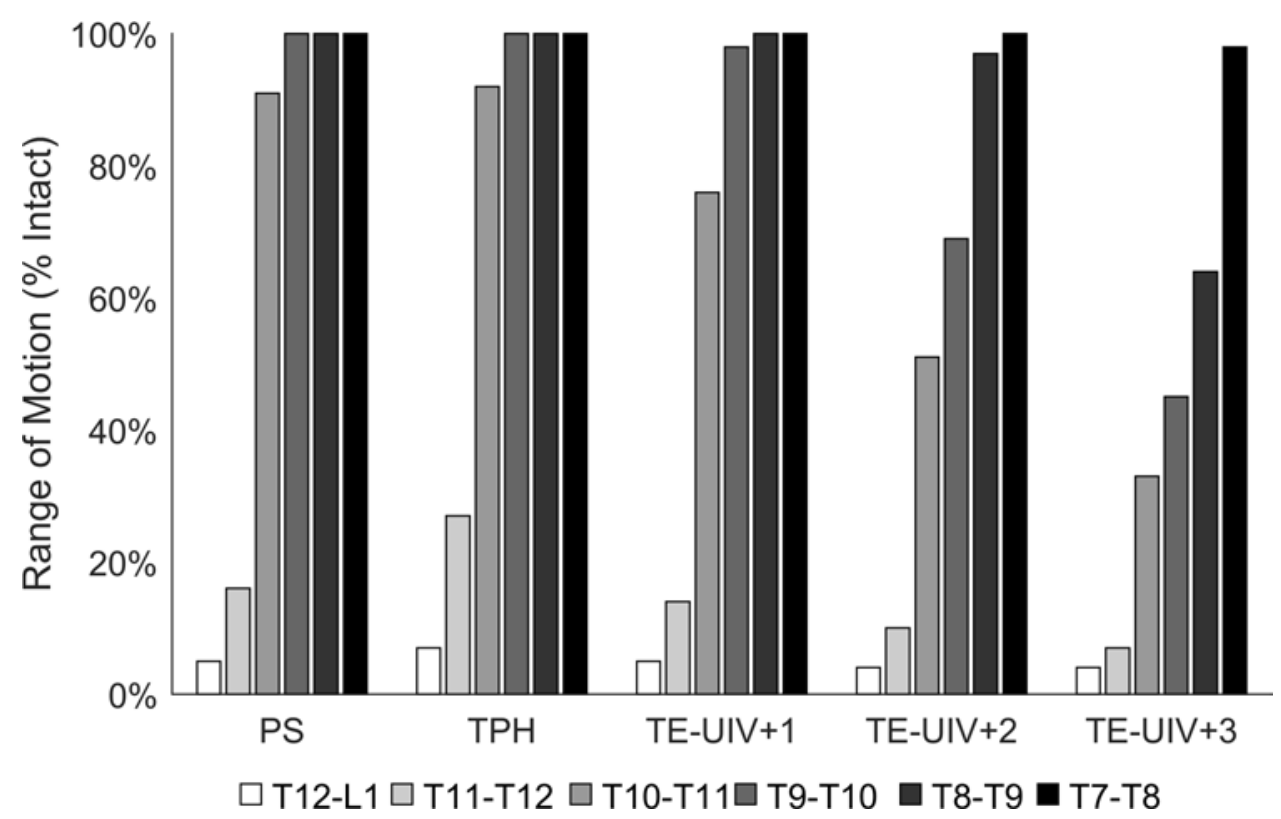

FIG. 2. Range of motion (\% of ROM in intact model) by level for models tested with bilateral segmental pedicle screws and rod from T-11 to L-5 (PS); transverse process hooks at T-11 with segmental, bilateral pedicle screws from T-12 to L-5 (TPH); 1-level tethers anchored from the UIV to UIV+1 (TE-UIV+1); 2-level tethers anchored from the UIV to UIV+1 and UIV+2 (TE-UIV+2); and 3-level tethers anchored from the UIV to UIV+1, UIV+2, and UIV+3 (TE-UIV+3).

of intact at T10-11. Intradiscal pressure for the TPH model demonstrated a more gradual transition from T11-12 (47\% of intact) to T10-11 (100\% of intact). Posterior tethered constructs demonstrated further dampening of intradiscal pressures at the proximal terminus of the constructs, as intradiscal pressures for TE-UIV+1 were $88 \%$ of intact at T10-11 and $100 \%$ of intact at T9-10. Intradiscal pressures for TE-UIV+2 was $69 \%$ of intact at T10-11, 81\% at T9-10, and $100 \%$ of intact at T8-T9. Intradiscal pressures for TE$\mathrm{UIV}+3$ were $55 \%$ of intact at $\mathrm{T} 10-11,65 \%$ at $\mathrm{T} 9-10$, and $78 \%$ of intact at T8-T9 (Fig. 3).

\section{Pedicle Screw Forces}

The left and right pedicle screw forces were measured and averaged at each level. The PS model demonstrated the largest screw forces, with $54.2 \mathrm{~N}$ at T-11. Adding tethers reduced the force on the screws at the UIV by distributing forces to the screws on the proximal vertebrae. Screw forces for the TE-UIV+1 model were $41.2 \mathrm{~N}$ at T-11 and $12.1 \mathrm{~N}$ at T-10. Screw forces for the TE-UIV+2 model were $23.6 \mathrm{~N}$ at T-11, 5.0 $\mathrm{N}$ at T-10, and $12.1 \mathrm{~N}$ at T-9. Screw forces for the TE-UIV+3 model were $11.6 \mathrm{~N}$ at T-11, 2.2 $\mathrm{N}$ at T-10, $6.0 \mathrm{~N}$ at T-9, and $21.6 \mathrm{~N}$ at T-8. The TPH model resulted in increased screw forces in all segments below the UIV, with $21.8 \mathrm{~N}$ at T-12, $6.4 \mathrm{~N}$ at L-1, and $2.8 \mathrm{~N}$ at L-2 (Fig. 4).

\section{Posterior Ligament Complex Forces}

The ISL/SSL complex forces were measured at each level and normalized to the intact condition. For the PS model, the ISL/SSL forces increased from $4 \%$ of intact at the T11-12 segment to $100 \%$ of intact at the T10-11 segment. ISL/SSL forces for TPH were $59 \%$ of intact at
T11-12, and $100 \%$ at T10-11. ISL/SSL forces for TEUIV+1 were $3 \%$ of intact at T11-12, $82 \%$ at T10-11, and $100 \%$ at T9-10. ISL/SSL for TE-UIV+2 were $2 \%$ of intact at $\mathrm{T} 11-12,53 \%$ at $\mathrm{T} 10-11,66 \%$ at $\mathrm{T} 9-10$, and $100 \%$ at T8-T9. ISL/SSL forces for TE-UIV+3 were $2 \%$ of intact at $\mathrm{T} 11-12,33 \%$ at $\mathrm{T} 10-11,43 \%$ at $\mathrm{T} 9-10,57 \%$ at $\mathrm{T} 8-9$, and $100 \%$ at T7-T8 (Fig. 5).

\section{Discussion}

PJK remains problematic following multilevel spine surgery and can have a substantial impact on patient morbidity as well as the financial costs of spine surgery., ${ }^{9,15,16,27}$ The failure modes and risk factors for PJK are multifactorial and not fully understood., ${ }^{5,6,14,15}$ The pathoanatomical modes of failure that lead to acute PJK can be generally categorized as either soft tissue of hard tissue failure, with soft tissue failures typically occurring in the upper thoracic region and hard tissue failures occurring in the thoracolumbar region. ${ }^{13,29}$ In a retrospective study of 1218 patients treated for adult spinal deformity, Hostin et al. identified 68 cases (5.6\%) that resulted in proximal junctional failure (PJF). Failures most often occurred in the thoracolumbar region, with $66 \%$ of patients experiencing failure compared with $34 \%$ experiencing upper thoracic failure..$^{13}$ Annis et al. ${ }^{2}$ had similar findings, concluding that the incidence of PJF is high if the UIV is in the lower thoracic or lumbar spine, trending toward higher rates when the UIV is at T-10. A number of studies have investigated and identified a number of risk factors for PJK, including surgical approach, type of instrumentation, amount of preoperative malalignment, amount of deformity correction, posterior ligament complex disruption, fusion to the sacrum, adjacent segment disc degeneration, older age, 


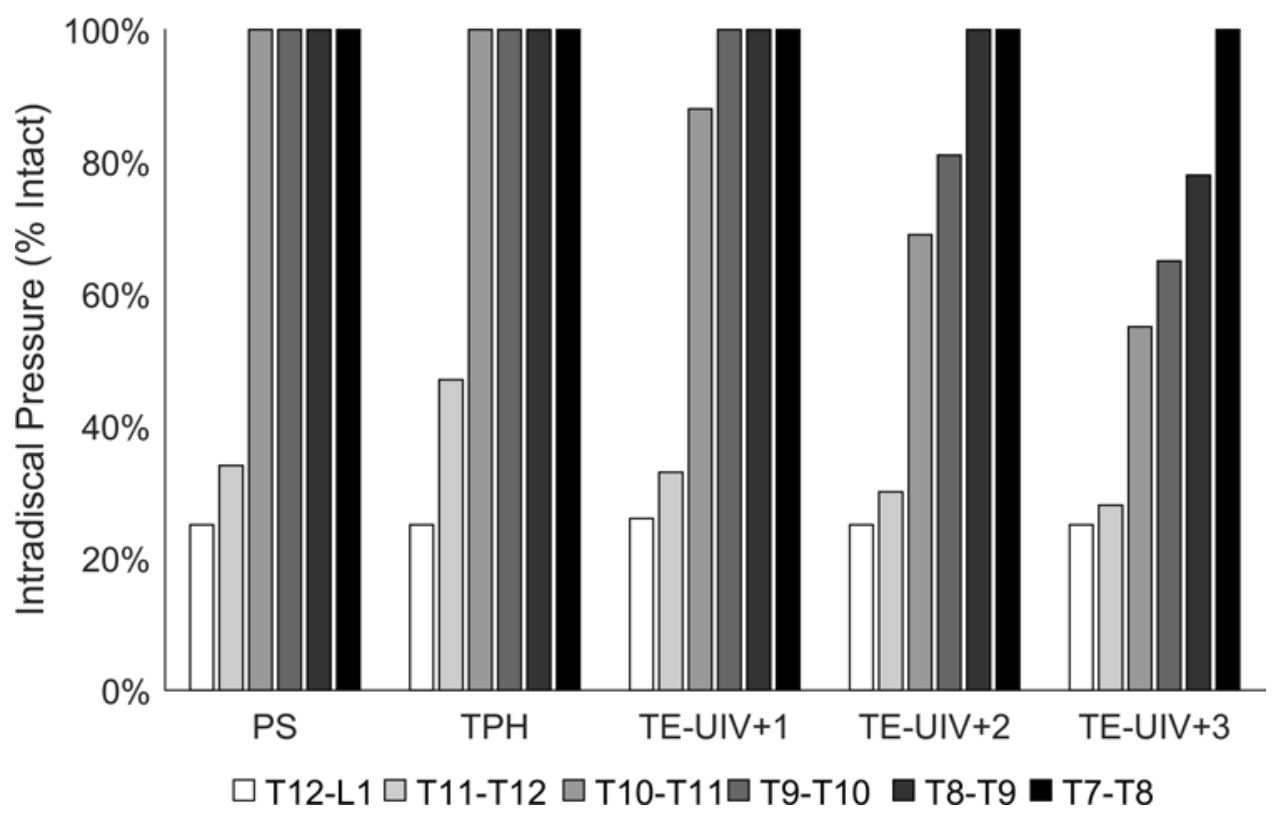

FIG. 3. Intradiscal pressures (\% of pressure in intact model) by level for models tested with bilateral segmental pedicle screws and rod from T-11 to L-5 (PS); transverse process hooks at T-11 with segmental, bilateral pedicle screws from T-12 to L-5 (TPH); 1-level tethers anchored from the UIV to UIV+1 (TE-UIV+1); 2-level tethers anchored from the UIV to UIV+1 and UIV+2 (TE-UIV+2); and 3-level tethers anchored from the UIV to UIV+1, UIV+2, and UIV+3 (TE-UIV+3).

osteoporosis, and construct rigidity.5,6,9,26-28 Consequently, several different surgical strategies have been proposed to mitigate the occurrence of PJK, including preservation of the posterior ligament complex and adjacent facet joints, use of vertebral augmentation with polymethyl methacrylate (PMMA) to prevent vertebral compression fracture, and use of less rigid fixation at the proximal terminus of the construct, including transition rods, TPHs, and/or dynamic stabilization techniques. ${ }^{4,25}$

In the current study, we investigated if a surgical strategy using posterior tethers could aid in mitigating the risk for PJF by dampening the proximal transition forces from

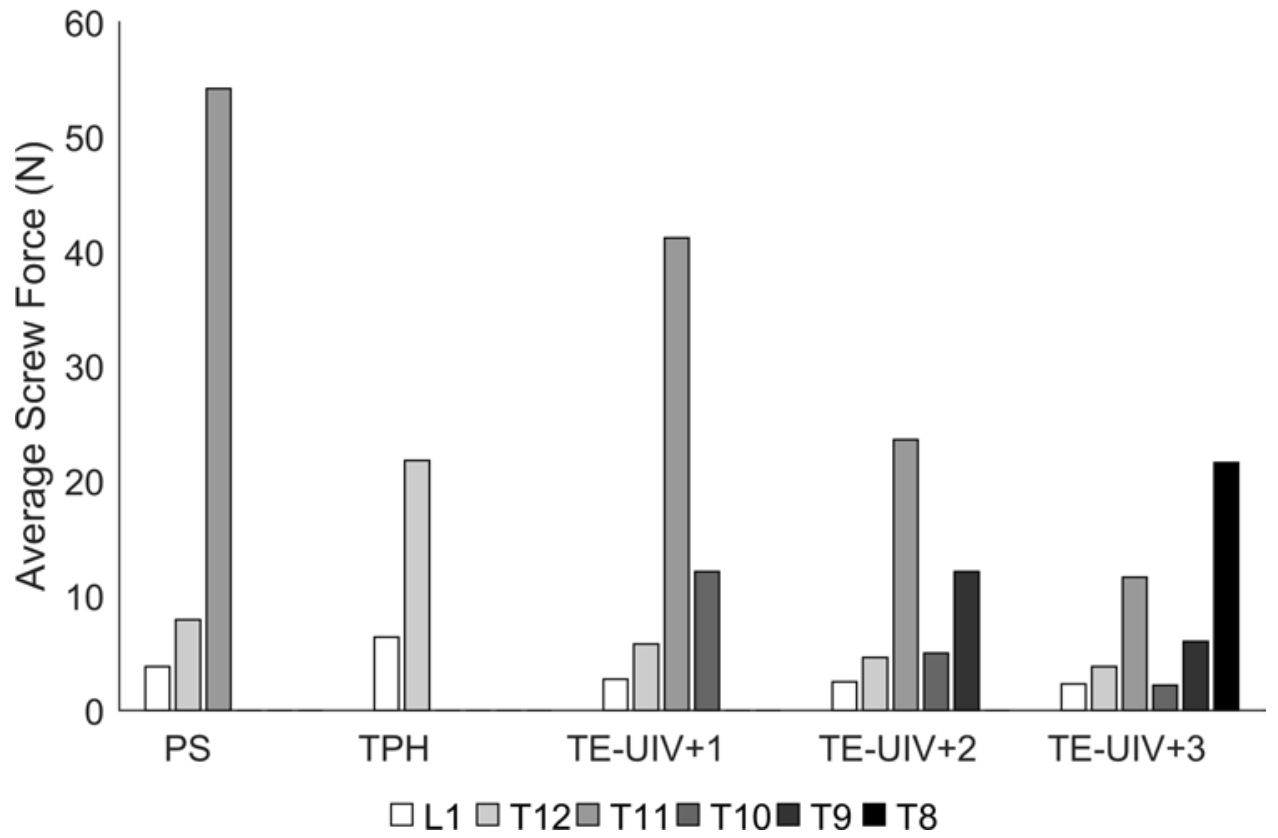

FIG. 4. Screw loads by level between T-8 and L-1 for models tested with bilateral segmental pedicle screws and rod from T-11 to L-5 (PS); transverse process hooks at T-11 with segmental, bilateral pedicle screws from T-12 to L-5 (TPH); 1-level tethers anchored from the UIV to UIV+1 (TE-UIV+1); 2-level tethers anchored from the UIV to UIV+1 and UIV+2 (TE-UIV+2); and 3-level tethers anchored from the UIV to UIV+1, UIV+2, and UIV+3 (TE-UIV+3). 


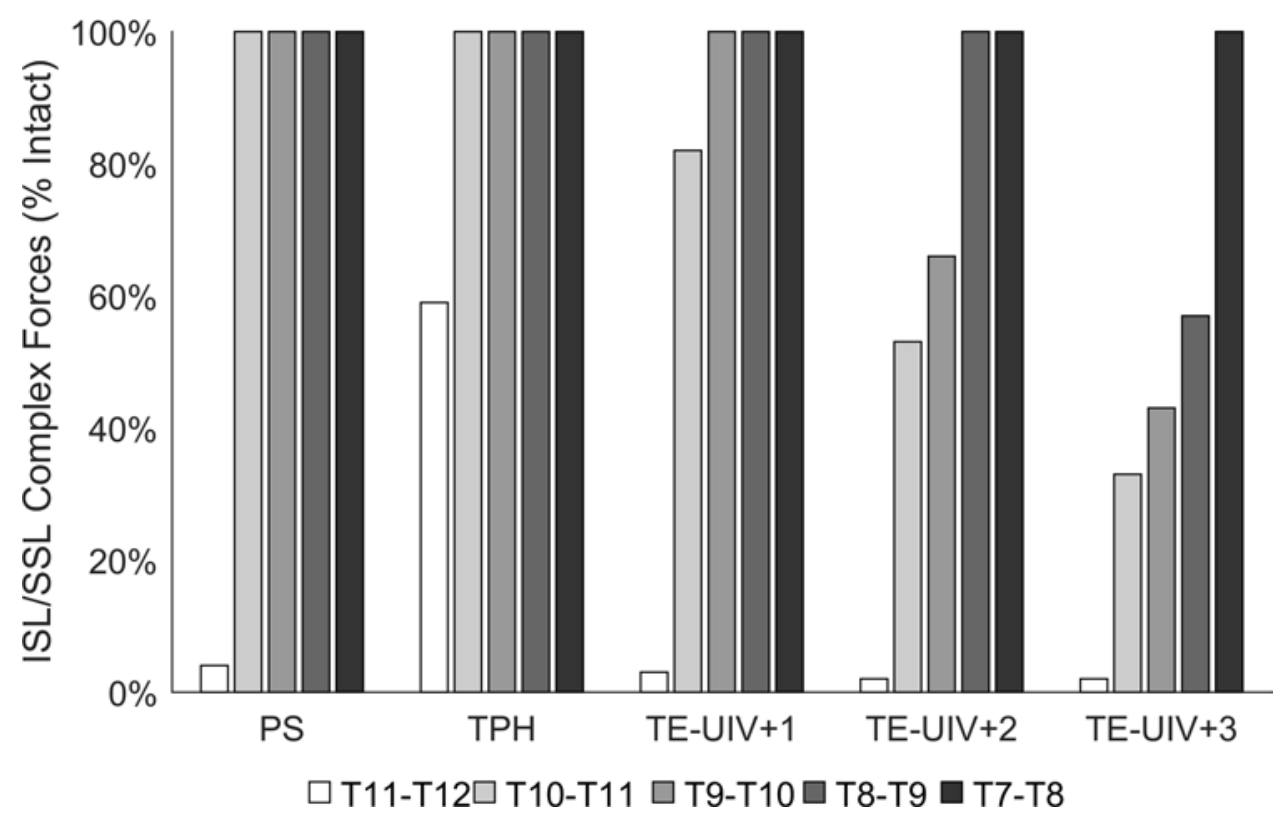

FIG. 5. ISL/SSL complex forces (\% of force in intact model) for models tested with bilateral segmental pedicle screws and rod from T-11 to L-5 (PS); transverse process hooks at T-11 with segmental, bilateral pedicle screws from T-12 to L-5 (TPH); 1-level tethers anchored from the UIV to UIV+1 (TE-UIV+1); 2-level tethers anchored from the UIV to UIV+1 and UIV+2 (TE-UIV+2); and 3-level tethers anchored from the UIV to UIV+1, UIV+2, and UIV+3 (TE-UIV+3).

the instrumented to the uninstrumented segments. The results of our finite element analysis indicated that the addition of posterior column based elastic tethers that extend cranially from the UIV and are anchored to the adjacent vertebrae create a more gradual transition to normal range of motion at the cranial vertebral segments than standalone bilateral pedicle screws or transverse process hooks. Consequently, the use of proximal posterior tethers may be a viable solution to help limit the occurrence of PJK. We used a finite element model rather than cadaveric testing due to limitations of accurately measuring and comparing construct forces in a cadaver model. Finite element analysis allows for estimation of intradiscal pressure, screw forces, and ligament forces that cannot be easily measured experimentally. This is consistent with recommendations by Cahill et al., ${ }^{4}$ who noted that cadaveric tests require several loading cycles for each test condition to generate ROM data. As a result, evaluation of the differences in construct rigidity would be confounded by cadaveric soft tissue and implant-bone interface degradation, particularly with a large number of test conditions. Use of a finite element model allows for direct comparison of test conditions by avoiding specimen variability and degradation inherent to cadaveric tests.

A number of ex vivo studies have investigated strategies to define the biomechanical factors that may contribute to the development of PJK and therefore help mitigate the occurrence of PJK. Anderson et al. ${ }^{1}$ studied the impact of dissecting posterior skeletal tissue at the UIV. They found the posterior skeletal structures of the thoracic spine that are often exposed during dissection for UIV instrumentation can decrease the flexion stiffness of the upper adjacent motion segment. Removal of the SSL and ISL created a significant loss of flexion stability as the tethering effect of these ligaments was lost. Cammarata et al. ${ }^{5}$ developed computational musculoskeletal models to study the impact of proximal soft tissue dissection, UIV implant type, sagittal rod curvature, and the use of bilateral transition rods on biomechanical properties of the proximal spine. They demonstrated that use of transverse process hooks at the UIV (rather than the use of pedicle screws at the UIV) and the use of tapered 3.5- to 5.5-mm transition rods at the UIV (rather than a continuous 5.5-mmdiameter rod at the UIV) allow for decreased rigidity at the UIV and reduced biomechanical risk for PJK. They also found that preserving the posterior structures at the UIV may decrease the risk for PJK. Cahill et al. ${ }^{4}$ used a finite element model of a healthy thoracic segment to determine whether a tapered rod would prevent PJK by enabling a gradual transition to normal biomechanics. Their model indicated that a $3.5-$ to $5.5-\mathrm{mm}$ transition rod at the UIV+1 terminus of a T3-12 pedicle screw construct decreases the terminal forces that potentially lead to PJK. They also demonstrated the importance of the ISL/SSL complex in providing flexion stability. Disc pressure and angular displacement increased for levels above the UIV when the ISL/SSL complex was removed. In a porcine model, Thawrani et al. ${ }^{25}$ demonstrated that transverse process hooks at the UIV provided a more gradual transition to normal motion compared with pedicle screws in long posterior spinal fusion constructs. This study did not address the effect of an initial deformity, the use of implants, or disc degeneration that may develop over time. In each of these ex vivo studies, the effect of the rib cage on motion segment stiffness was excluded. These studies used physiological loads to assess the change in spinal rigidity across 
the UIV and demonstrated that rigid fixation at the UIV creates a large change in stiffness as the spine transitions from the instrumented to the uninstrumented spine. This differential in stiffness between the UIV and UIV+1 is believed to be a biomechanical factor for development of PJK. Consequently, occurrence of PJK might be reduced by using less rigid constructs at the proximal terminus of the instrumentation.

Similar to Thawarani et al., we found that transverse process hooks at the UIV provided a small change in transitional ROM compared with rigid fixation. However, the addition of posterior tethers cranial to the UIV created a significantly more gradual transition to normal motion, especially when tethers were implanted across multiple levels (UIV+2 and UIV+3). The tethers provided a stabilizing structure as the spine transitioned away from rigid fixation. We found that distribution of the forces over several segments through the use of multilevel posterior tethers may protect against vertebral compression fractures and screw pullout, as the model demonstrated that pedicle screw forces at the UIV were decreased when posterior tethers were added to the construct. It is possible that through the use of posterior tethers, the decreased load at the UIV might have the advantageous effect of creating a "soft landing" zone for the forces on the uninstrumented vertebrae and might thereby reduce adjacent-segment loads imparted to the inferior endplate of the UIV+1, thus reducing the risk of endplate fracture at the UIV+1.

The posterior ligament complex via the ISL/SSL acts as a primary constraint to pathological flexion forces. Cahill et al. ${ }^{4}$ demonstrated that forward angular displacement and disc pressure increased when the ISL/SSL complex was removed above the UIV. Disc pressures also increased with posterior ligament resection. In a retrospective study of 174 cases, Yagi et al. ${ }^{27}$ also suggested that preserving the posterior ligament structure was important in preventing the incidence of PJK. Our model demonstrated that the posterior tethers played a similar role to the posterior ligament complex by limiting forward angular displacement. We found that posterior tethers shared load with the ISL/SSL complex, as demonstrated by the reduction of the forces measured in the posterior ligaments when using the posterior tethers cranial to the UIV. Disc pressures similarly decreased when tethers were added.

There are several important limitations to our data and this study. It is critical for the reader to understand that finite element analysis does not account for the variability of an anisotropic system, such as a biological model. We have attempted to investigate the ability of posterior polyester tethers to distribute loads at the vertebral segments in a long instrumented spine construct; however, finite element analysis is unable to model the variable stresses that occur on the implants and the anatomy in a true biological system. Additionally, finite element analysis cannot account for the variability of the anatomy and associated implant and anatomical stresses that occur in the individual patient. Therefore, these data cannot be used to predict which patients are at risk for PJK. Furthermore, while our data demonstrated differences in adjacent-segment ROM, intradiscal pressure, and implant and posterior ligament forces between the PS, TPH, and posterior tether constructs, the clinical applicability of these differences is unknown. With respect to modeling for finite element analysis, the model used in this study excluded the ribs and therefore does not account for segment stiffness in the spinal column due to the rib cage. The model used in this study also does not account for external muscle forces due to the complexity required to implement them. This model also does not account for upper-body weight and the subsequent loads exerted on the spinal column. Consequently, no direct clinical conclusions can be made from these data, and further testing and clinical studies are needed to investigate the ability of posterior tethers to reduce PJK and to delineate the appropriate patient for use. Thus a clinical trial is necessary to confirm the finite element model findings from this study. We also acknowledge that the surgical addition of posterior tethers requires additional tissue dissection, and care must be taken to avoid damaging the ISL/SSL complex. Minimally invasive techniques could be employed to minimize any disruption. The potential benefit of adding tethers may outweigh the drawback of additional tissue dissection.

Despite the limitations inherent to finite element models, this study demonstrated the biomechanical effects of posterior elastic tethers on spinal segment stiffness. Finite element analysis provided the ability to make a direct biomechanical comparison between different constructs. Adding additional anatomical structures such as the rib cage to the model may provide a more anatomical model, but the relative differences between constructs (i.e., ranking of results) are expected to remain consistent. Future work on the use of posterior tethers to reduce PJK in long instrumented spine constructs must include a clinical trial to evaluate the outcomes of patients treated with posterior tethers. The next steps for this research will include evaluation of the incidence of PJK in a group of patients treated with posterior tethers in conjunction with multilevel posterior spine constructs, followed by a prospective comparative analysis of patients treated with and without posterior tethers in multilevel fusion. This clinical information will be critical to the ability to accurately comment on the clinical applicability of posterior tethers to reduce PJK in multilevel spine fusion procedures.

\section{Conclusions}

Distribution of the forces at and above the UIV in long, instrumented spine constructs may decrease the biomechanical risk of postoperative PJF. We used finite element analysis to demonstrate that augmenting a long pedicle screw construct in the thoracic and lumbar spine with posterior polyester tethers created a more gradual transition in ROM, forces on the pedicle screws, and forces in the posterior spinal ligaments from the instrumented to uninstrumented spinal segments, compared with constructs that used PS or TPH at the UIV. These results suggest that the use of posterior tethers in conjunction with multilevel instrumented spinal constructs may help address the biomechanical risk factors associated with PJK. Further research, including a clinical trial evaluating the use of posterior tethers in multilevel posterior fusion, is needed to delineate the clinical applicability of these findings. 


\section{References}

1. Anderson AL, McIff TE, Asher MA, Burton DC, Glattes RC: The effect of posterior thoracic spine anatomical structures on motion segment flexion stiffness. Spine (Phila Pa 1976) 34:441-446, 2009

2. Annis P, Lawrence BD, Spiker WR, Zhang Y, Chen W, Daubs $\mathrm{MD}$, et al: Predictive factors for acute proximal junctional failure after adult deformity surgery with upper instrumented vertebrae in the thoracolumbar spine. Evid Based Spine Care J 5:160-162, 2014

3. Bowden AE, Guerin HL, Villarraga ML, Patwardhan AG, Ochoa JA: Quality of motion considerations in numerical analysis of motion restoring implants of the spine. Clin Biomech (Bristol, Avon) 23:536-544, 2008

4. Cahill PJ, Wang W, Asghar J, Booker R, Betz RR, Ramsey C, et al: The use of a transition rod may prevent proximal junctional kyphosis in the thoracic spine after scoliosis surgery: a finite element analysis. Spine (Phila Pa 1976) 37:E687E695, 2012

5. Cammarata M, Aubin CE, Wang X, Mac-Thiong JM: Biomechanical risk factors for proximal junctional kyphosis: a detailed numerical analysis of surgical instrumentation variables. Spine (Phila Pa 1976) 39:E500-E507, 2014

6. Denis F, Sun EC, Winter RB: Incidence and risk factors for proximal and distal junctional kyphosis following surgical treatment for Scheuermann kyphosis: minimum five-year follow-up. Spine (Phila Pa 1976) 34:E729-E734, 2009

7. Fujimori T, Inoue S, Le H, Schairer WW, Berven SH, Tay $\mathrm{BK}$, et al: Long fusion from sacrum to thoracic spine for adult spinal deformity with sagittal imbalance: upper versus lower thoracic spine as site of upper instrumented vertebra. Neurosurg Focus 36(5):E9, 2014

8. Fujiwara A, Lim TH, An HS, Tanaka N, Jeon CH, Andersson $\mathrm{GB}$, et al: The effect of disc degeneration and facet joint osteoarthritis on the segmental flexibility of the lumbar spine. Spine (Phila Pa 1976) 25:3036-3044, 2000

9. Glattes RC, Bridwell KH, Lenke LG, Kim YJ, Rinella A, Edwards C II: Proximal junctional kyphosis in adult spinal deformity following long instrumented posterior spinal fusion: incidence, outcomes, and risk factor analysis. Spine (Phila Pa 1976) 30:1643-1649, 2005

10. Goel VK, Monroe BT, Gilbertson LG, Brinckmann P: Interlaminar shear stresses and laminae separation in a disc. Finite element analysis of the L3-L4 motion segment subjected to axial compressive loads. Spine (Phila Pa 1976) 20:689-698, 1995

11. Guan Y, Yoganandan N, Zhang J, Pintar FA, Cusick JF, Wolfla CE, et al: Validation of a clinical finite element model of the human lumbosacral spine. Med Biol Eng Comput 44:633-641, 2006

12. Hart RA, McCarthy I, Ames CP, Shaffrey CI, Hamilton DK, Hostin R: Proximal junctional kyphosis and proximal junctional failure. Neurosurg Clin N Am 24:213-218, 2013

13. Hostin R, McCarthy I, O'Brien M, Bess S, Line B, BoachieAdjei $\mathrm{O}$, et al: Incidence, mode, and location of acute proximal junctional failures after surgical treatment of adult spinal deformity. Spine (Phila Pa 1976) 38:1008-1015, 2013

14. Kim HJ, Lenke LG, Shaffrey CI, Van Alstyne EM, Skelly AC: Proximal junctional kyphosis as a distinct form of adjacent segment pathology after spinal deformity surgery: a systematic review. Spine (Phila Pa 1976) 37 (22 Suppl):S144S164, 2012

15. Kim YJ, Bridwell KH, Lenke LG, Kim J, Cho SK: Proximal junctional kyphosis in adolescent idiopathic scoliosis following segmental posterior spinal instrumentation and fusion: minimum 5-year follow-up. Spine (Phila Pa 1976) 30:20452050, 2005

16. Mendoza-Lattes S, Ries Z, Gao Y, Weinstein SL: Proximal junctional kyphosis in adult reconstructive spine surgery re- sults from incomplete restoration of the lumbar lordosis relative to the magnitude of the thoracic kyphosis. Iowa Orthop J 31:199-206, 2011

17. Morgan EF, Bayraktar HH, Keaveny TM: Trabecular bone modulus-density relationships depend on anatomic site. $\mathbf{J}$ Biomech 36:897-904, 2003

18. Niosi CA, Zhu QA, Wilson DC, Keynan O, Wilson DR, Oxland TR: Biomechanical characterization of the three-dimensional kinematic behaviour of the Dynesys dynamic stabilization system: an in vitro study. Eur Spine J 15:913-922, 2006

19. Panjabi MM: Hybrid multidirectional test method to evaluate spinal adjacent-level effects. Clin Biomech (Bristol, Avon) 22:257-265, 2007

20. Panjabi MM, Abumi K, Duranceau J, Crisco JJ: Biomechanical evaluation of spinal fixation devices: II. Stability provided by eight internal fixation devices. Spine (Phila Pa 1976) 13:1135-1140, 1988

21. Schairer WW, Carrer A, Deviren V, Hu SS, Takemoto S, Mummaneni P, et al: Hospital readmission after spine fusion for adult spinal deformity. Spine (Phila Pa 1976) 38:16811689,2013

22. Schilling C, Krüger S, Grupp TM, Duda GN, Blömer W, Rohlmann A: The effect of design parameters of dynamic pedicle screw systems on kinematics and load bearing: an in vitro study. Eur Spine J 20:297-307, 2011

23. Schmidt H, Heuer F, Drumm J, Klezl Z, Claes L, Wilke HJ: Application of a calibration method provides more realistic results for a finite element model of a lumbar spinal segment. Clin Biomech (Bristol, Avon) 22:377-384, 2007

24. Schmoelz W, Huber JF, Nydegger T, Claes L, Wilke HJ: Dynamic stabilization of the lumbar spine and its effects on adjacent segments: an in vitro experiment. J Spinal Disord Tech 16:418-423, 2003

25. Thawrani DP, Glos DL, Coombs MT, Bylski-Austrow DI, Sturm PF: Transverse process hooks at upper instrumented vertebra provide more gradual motion transition than pedicle screws. Spine (Phila Pa 1976) 39:E826-E832, 2014

26. Watanabe K, Lenke LG, Bridwell KH, Kim YJ, Koester L, Hensley M: Proximal junctional vertebral fracture in adults after spinal deformity surgery using pedicle screw constructs: analysis of morphological features. Spine (Phila Pa 1976) 35:138-145, 2010

27. Yagi M, Akilah KB, Boachie-Adjei O: Incidence, risk factors and classification of proximal junctional kyphosis: surgical outcomes review of adult idiopathic scoliosis. Spine (Phila Pa 1976) 36:E60-E68, 2011

28. Yagi M, King AB, Boachie-Adjei O: Incidence, risk factors, and natural course of proximal junctional kyphosis: surgical outcomes review of adult idiopathic scoliosis. Minimum 5 years of follow-up. Spine (Phila Pa 1976) 37:1479-1489, 2012

29. Yagi M, Rahm M, Gaines R, Maziad A, Ross T, Kim HJ, et al: Characterization and surgical outcomes of proximal junctional failure in surgically treated patients with adult spinal deformity. Spine (Phila Pa 1976) 39:E607-E614, 2014

\section{Disclosures}

Research materials and support were provided by NuVasive, Inc. Dr. Bess reports a consultant relationship with K2 Medical and AlloSource; a patent holder relationship with Innovasis and K2 Medical; receipt of support for study described from NuVasive; and support of non-study related efforts from DePuy Spine, Innovasis, K2 Medical, Stryker, and Medtronic. Mr. Harris and Dr. Turner report employee relationships with and direct stock ownership in NuVasive. Dr. Lafage reports an ownership interest in Nemaris Inc.; a consultant relationship with NuVasive; speaking/ teaching arrangements with DePuy, Medicrea, and NuVasive; and 
support of non-study related efforts from SRS, NIH, and DePuy (paid through ISSGF). Dr. Smith reports consultant relationships with Zimmer Biomet, NuVasive, and Cerapedics; receipt of support for study described from NuVasive; support of non-study related effort from DePuy Synthes; and fellowship grant support from NREF and AOSpine. Dr. Shaffrey reports a consultant relationship with Zimmer Biomet, Medtronic, NuVasive, K2M, and Stryker; direct stock ownership in NuVasive; and a patent holder relationship with Zimmer Biomet, Medtronic, and NuVasive. Dr. Schwab reports direct stock ownership in Nemaris Inc.; consultant and teaching/speaking relationships with K2M, NuVasive, Zimmer Biomet, MSD, and Medicrea; and support of non-study related effort from SRS, AO, and DePuy Spine Synthes (paid through ISSGF). Dr. Haid reports a consultant relationship with NuVasive, K2 Medical, and AlloSource; a patent holder relationship with K2 Medical and Innovasis; support for the study described from NuVasive; and support of non-study related efforts from DePuy Synthes, K2 Medical, Medtronic, Biomet, and Stryker.

\section{Author Contributions}

Conception and design: Bess, Harris. Acquisition of data: Harris. Analysis and interpretation of data: all authors. Drafting the article: Bess, Harris. Critically revising the article: Bess, Harris, Turner, Lafage, Smith, Shaffrey. Reviewed submitted version of manuscript: Bess, Harris, Smith, Shaffrey, Schwab, Haid. Approved the final version of the manuscript on behalf of all authors: Bess. Administrative/technical/material support: Harris, Turner, Lafage. Study supervision: Bess.

\section{Correspondence}

Shay Bess, Hospital for Joint Diseases, 301 East 17th St., Fourth Fl., Spine Center, New York, NY 10003. email: shay_bess@ hotmail.com. 